Bull. Austral. Math. Soc.

$34 \mathrm{C} 10,34 \mathrm{~K} 40$

VOL. 56 (1997) [1-16]

\title{
CONDITIONS FOR OSCILLATION OF FIRST ORDER NEUTRAL DELAY DIFFERENTIAL EQUATIONS
}

\section{ZIWEN JIANG}

In this paper, some new sufficient conditions for oscillation of first order neutral delay differential equations with several variable coefficients are obtained. These sufficient conditions include and are in many cases weaker than those known.

\section{INTRODUCTION}

The oscillation theory of first order neutral delay differential equations (NDDEs for short) has been extensively developed during the past few years. We refer to the works of Grammatikopoulos et al [1, 2, 3], Ladas and Sficas [4], Gopalsamy and Zhang [5], Jiang Ziwen [6] for some results related to the oscillations of NDDEs. Recently, there has been some interest in the oscillation theory of first order NDDEs when the NDDE has one or more variable coefficients (for example see Gopalsamy and Zhang [5], Jiang Ziwen [6]).

In this paper, we study the oscillation of first order NDDEs with several variable coefficients

$$
\frac{\mathrm{d}}{\mathrm{dt}}\left(x(t)-\sum_{i=1}^{m} c_{i} x\left(t-r_{i}\right)\right)+\sum_{j=1}^{n} p_{j}(t) x\left(t-s_{j}\right)=0
$$

and

$$
\frac{\mathrm{d}}{\mathrm{dt}}\left(x(t)-\sum_{i=1}^{m} c_{i}(t) x\left(t-r_{i}\right)\right)+\sum_{j=1}^{n} p_{j}(t) x\left(t-s_{j}\right)=0 .
$$

When $m=n=1$, Gopalsamy and Zhang in [5], Jiang Ziwen in [6] obtained some sufficient conditions for oscillation of the first order NDDEs (1.1) and (1.2). But these sufficient conditions in [5] or [6] are strong. The purpose of this paper is to give some new sufficient conditions which are weaker than those in [5] and [6] for oscillation of the first order NDDEs (1.1) and (1.2). In order to achieve this aim, we first obtain some new sufficient conditions for oscillation of the first order NDDEs with several coefficients

$$
\frac{\mathrm{d}}{\mathrm{dt}}\left(x(t)-\sum_{i=1}^{m} c_{i} x\left(t-r_{i}\right)\right)+\sum_{j=1}^{n} p_{j} x\left(t-s_{j}\right)=0 .
$$

Received 9th July, 1996

Copyright Clearance Centre, Inc. Serial-fee code: 0004-9729/97 \$A2.00+0.00. 
Then we use these new sufficient conditions for oscillation of (1.3) and two Lemmas to derive some new sufficient conditions for oscillation of (1.1) and (1.2). All of these new sufficient conditions we obtain include and are in many cases weaker than those in the articles $[\mathbf{2}, \mathbf{3}, \mathbf{4}, \mathbf{5}, \mathbf{6}]$; furthermore these new sufficient conditions can be verified when the coefficients of NDDEs are given. That is, these sufficient conditions have relevance to the coefficients of NDDEs only.

\section{Lemmas AND Theorems}

In this section, we shall prove some lemmas and theorems which are the foundation of our main results. First, we consider the first order NDDEs

$$
\frac{\mathrm{d}}{\mathrm{dt}}\left(x(t)-\sum_{i=1}^{m} c_{i} x\left(t-r_{i}\right)\right)+\sum_{j=1}^{n} p_{j} x\left(t-s_{j}\right)=0
$$

where the coefficients satisfy

$$
\begin{aligned}
& c_{i}>0, i=1,2, \cdots, m, \sum_{i=1}^{m} c_{i}<1 \quad \text { and } \quad 0<r_{1} \leqslant r_{2} \leqslant \cdots \leqslant r_{m} \\
& p_{j}>0, j=1,2, \cdots, n \text { and } 0<s_{1} \leqslant s_{2} \leqslant \cdots \leqslant s_{n} .
\end{aligned}
$$

We have the following result:

LEMMA 2.1. Assume that

$$
\sum_{i=1}^{m} c_{i} \exp \left(v r_{i}\right)+\sum_{j=1}^{n} p_{j} \exp \left(v s_{j}\right) / v>1 \text { for all } v>0
$$

Then all solutions of (1.3) are oscillatory.

PROOF: The characteristic equation of (1.3) is

$$
f(\lambda)=\lambda-\lambda \sum_{i=1}^{m} c_{i} \exp \left(-\lambda r_{i}\right)+\sum_{j=1}^{n} p_{j} \exp \left(-\lambda s_{j}\right)=0
$$

To prove this result, it suffices to prove that (2.4) has no real roots under the assumption (2.1) and (2.2) (see [1, Theorem]). We note that any real root of (2.4) cannot be positive under the assumption (2.1) and (2.2). Since $f(0)=\sum_{j=1}^{n} p_{j}>0, \lambda=0$ is not a root of (2.4). Thus any real root of (2.4) can only be negative. Let $\lambda=-v_{0}<0$ be a root of (2.4), then

$$
1-\sum_{i=1}^{m} c_{i} \exp \left(v_{0} r_{i}\right)-\sum_{j=1}^{n} p_{j} \exp \left(v_{0} s_{j}\right) / v_{0}=0, v_{0}>0
$$


which contradicts the condition (2.3). Hence (2.4) has no real roots under the assumption (2.1) and (2.2). The proof is complete.

THEOREM 2.2. Assume that

$$
e \sum_{j=1}^{n} p_{j} s_{j} \geqslant 1-\sum_{i=1}^{m} c_{i} \exp \left(r_{i} \sum_{j=1}^{n} p_{j} /\left(1-\sum_{i=1}^{m} c_{i}\right)\right) .
$$

Then all solutions of (1.3) are oscillatory.

Proof: We define the function $f$ so that

$$
f(v)=\sum_{j=1}^{n} p_{j} \exp \left(v s_{j}\right) / v+\sum_{i=1}^{m} c_{i} \exp \left(v r_{i}\right)-1, v>0 .
$$

Consider the value of $f$ at $v$ for $0<v \leqslant \sum_{j=1}^{n} p_{j} /\left(1-\sum_{i=1}^{m} c_{i}\right)$, then

$$
f(v)>\sum_{j=1}^{n} p_{j} / v+\sum_{i=1}^{m} c_{i}-1
$$

Hence $f(v)>0$ for all $v$ satisfying $0<v \leqslant \sum_{j=1}^{n} p_{j} /\left(1-\sum_{i=1}^{m} c_{i}\right)$.

Let us now consider the value of $f$ at $v$ for $v>\sum_{j=1}^{n} p_{j} /\left(1-\sum_{i=1}^{m} c_{i}\right)$ and note that $p_{j} \exp \left(v s_{j}\right) / v$ has a global minimum at $1 / s_{j}$ and the minimum value is $e p_{j} s_{j}$ for $j=1,2, \cdots, n$. Then

$$
f(v)>e \sum_{j=1}^{n} p_{j} s_{j}+\sum_{i=1}^{m} c_{i} \exp \left(r_{i} \sum_{j=1}^{n} p_{j} /\left(1-\sum_{i=1}^{m} c_{i}\right)\right)-1 .
$$

But by our assumption (2.5), e $\sum_{j=1}^{n} p_{j} s_{j}+\sum_{i=1}^{m} c_{i} \exp \left(r_{i} \sum_{j=1}^{n} p_{j} /\left(1-\sum_{i=1}^{m} c_{i}\right)\right)-1 \geqslant 0$ showing that $f(v)>0$ for all $v>\sum_{j=1}^{n} p_{j} /\left(1-\sum_{i=1}^{m} c_{i}\right)$.

Thus we have shown that (2.3) holds. It follows from Lemma 2.1 that all solutions of (1.3) are oscillatory and the proof is complete.

THEOREM 2.3. Assume that

(i) $\sum_{j=1}^{n} p_{j} s_{j} \geqslant 1-\sum_{i=1}^{m} c_{i}, \quad$ or 
(ii) $\sum_{j=1}^{n} p_{j} s_{j}<1-\sum_{i=1}^{m} c_{i}$ and

$$
e \sum_{j=1}^{n} p_{j} s_{j} \geqslant 1-\sum_{i=1}^{m} c_{i} \exp \left(r_{i} \sum_{j=1}^{n} p_{j} /\left(1-\sum_{i=1}^{m} c_{i}-\sum_{j=1}^{n} p_{j} s_{j}\right)\right)
$$

Then all solutions of (1.3) are oscillatory.

Proof: (i) Since

$$
e \sum_{j=1}^{n} p_{j} s_{j} \geqslant e\left(1-\sum_{i=1}^{m} c_{i}\right)>1-\sum_{i=1}^{m} c_{i} \exp \left(r_{i} \sum_{j=1}^{n} p_{j} /\left(1-\sum_{i=1}^{m} c_{i}\right)\right)
$$

by Theorem 2.2, all solutions of (1.3) are oscillatory.

(ii) We define the function $f$ as in the proof of Theorem 2.2. We first consider the value of $f$ at $v$ for $v>\sum_{j=1}^{n} p_{j} /\left(1-\sum_{i=1}^{m} c_{i}-\sum_{j=1}^{n} p_{j} s_{j}\right)$ and note that $p_{j} \exp \left(v s_{j}\right) / v$ has a global minimum at $1 / s_{j}$ and the minimum value is $e p_{j} s_{j}$ for $j=1,2, \cdots, n$. Then

$$
\begin{aligned}
f(v) & =\sum_{j=1}^{n} p_{j} \exp \left(v s_{j}\right) / v+\sum_{i=1}^{m} c_{i} \exp \left(v r_{i}\right)-1 \\
& >e \sum_{j=1}^{n} p_{j} s_{j}+\sum_{i=1}^{m} c_{i} \exp \left(r_{i} \sum_{j=1}^{n} p_{j} /\left(1-\sum_{i=1}^{m} c_{i}-\sum_{j=1}^{n} p_{j} s_{j}\right)\right)-1 .
\end{aligned}
$$

By our assumption (2.6),

$$
e \sum_{j=1}^{n} p_{j} s_{j}+\sum_{i=1}^{m} c_{i} \exp \left(r_{i} \sum_{j=1}^{n} p_{j} /\left(1-\sum_{i=1}^{m} c_{i}-\sum_{j=1}^{n} p_{j} s_{j}\right)\right)-1>0
$$

showing that $f(v)>0$ for all $v>\sum_{j=1}^{n} p_{j} /\left(1-\sum_{i=1}^{m} c_{i}-\sum_{j=1}^{n} p_{j} s_{j}\right)$.

Next we consider the value of $f$ at $v$ for $v$ satisfying

$$
0<v \leqslant \sum_{j=1}^{n} p_{j} /\left(1-\sum_{i=1}^{m} c_{i}-\sum_{j=1}^{n} p_{j} s_{j}\right)
$$

We have

$$
f(v)=\sum_{j=1}^{n} p_{j} \exp \left(v s_{j}\right) / v+\sum_{i=1}^{m} c_{i} \exp \left(v r_{i}\right)-1>\sum_{j=1}^{n} p_{j}\left(1+v s_{j}\right) / v+\sum_{i=1}^{m} c_{i}-1
$$


Hence $f(v)>0$ for all $v$ satisfying $0<v \leqslant \sum_{j=1}^{n} p_{j} /\left(1-\sum_{i=1}^{m} c_{i}-\sum_{j=1}^{n} p_{j} s_{j}\right)$.

Thus we have shown that (2.3) holds. By Lemma 2.1, all solutions of (1.3) are oscillatory and the proof is complete.

In general, the conditions (2.5) and (2.6) are difficult to verify. In the following, we give some sufficient conditions which are easier to verify than the conditions (2.5) and (2.6).

THEOREM 2.4. Assume that there exists a nonnegative integer $N$ satisfying

$$
e \sum_{j=1}^{n} p_{j} s_{j} \geqslant 1-\sum_{i=1}^{m} c_{i} \sum_{k=0}^{N}\left(\left(r_{i} \sum_{j=1}^{n} p_{j}\right) /\left(1-\sum_{i=1}^{m} c_{i}\right)\right)^{k} /(k !)
$$

Then all solutions of (1.3) are oscillatory.

ProOF: Since

$$
\sum_{i=1}^{m} c_{i} \sum_{k=0}^{N}\left(\left(r_{i} \sum_{j=1}^{n} p_{j}\right) /\left(1-\sum_{i=1}^{m} c_{i}\right)\right)^{k} /(k !)<\sum_{i=1}^{m} c_{i} \exp \left(\left(r_{i} \sum_{j=1}^{n} p_{j}\right) /\left(1-\sum_{i=1}^{m} c_{i}\right)\right)
$$

(2.5) holds. It follows from Theorem 2.2 that all solutions of (1.3) are oscillatory and the proof is complete.

THEOREM 2.5. Assume that $\sum_{j=1}^{n} p_{j} s_{j}<1-\sum_{i=1}^{m} c_{i}$, and that there exists a nonnegative integer $N$ satisfying

$$
e \sum_{j=1}^{n} p_{j} s_{j} \geqslant 1-\sum_{i=1}^{m} c_{i} \sum_{k=0}^{N}\left(\left(r_{i} \sum_{j=1}^{n} p_{j}\right) /\left(1-\sum_{i=1}^{m} c_{i}-\sum_{j=1}^{n} p_{j} s_{j}\right)\right)^{k} /(k !)
$$

Then all solutions of (1.3) are oscillatory.

Proof: Since

$$
\begin{aligned}
\sum_{k=0}^{N}\left(\left(r_{i} \sum_{j=1}^{n} p_{j}\right) /\left(1-\sum_{i=1}^{m} c_{i}-\sum_{j=1}^{n} p_{j} s_{j}\right)\right)^{k} /(k !) & \\
& <\exp \left(\left(r_{i} \sum_{j=1}^{n} p_{j}\right) /\left(1-\sum_{i=1}^{m} c_{i}-\sum_{j=1}^{n} p_{j} s_{j}\right)\right)
\end{aligned}
$$

(2.6) holds. It follows from Theorem 2.3 that all solutions of (1.3) are oscillatory and the proof is complete. 
REMARK. The sufficient conditions of Theorem 2.2.-2.5. for oscillation of first order NDDEs include and are in many cases weaker than those of $[2,3,4,5,6]$, so the results of Theorem 2.2.-2.5. develop the results of $[2,3,4,5,6]$. See Example 1 and Example 2 in Section 4 of this paper.

Second, we consider the first order NDDEs

$$
\frac{\mathrm{d}}{\mathrm{dt}}\left(x(t)-\sum_{i=1}^{m} c_{i} x\left(t-r_{i}\right)\right)+\sum_{j=1}^{n} p_{j}(t) x\left(t-s_{j}\right)=0
$$

where the coefficients satisfy

(i) $\quad c_{i}>0, i=1,2, \cdots, m, \sum_{i=1}^{m} c_{i}<1 ; 0<r_{1} \leqslant r_{2} \leqslant \cdots \leqslant r_{m} ; 0<s_{1} \leqslant$ $s_{2} \leqslant \cdots \leqslant s_{n}$.

(ii) $p_{j}(t)$ is continuous and $p_{j}(t) \geqslant q_{j} \geqslant 0, j=1,2, \cdots, n, t \in R, \sum_{j=1}^{n} q_{j}>0$.

Then we have the following result:

LEMmA 2.6. Assume that $y(t)$ is a nonoscillatory solution of (1.1). Then $\lim _{t \rightarrow \infty} y(t)=0$.

The proof of Lemma 2.6 is similar to the proof of Lemma 2.7 , so we omit it.

Finally, we consider the first order NDDEs

$$
\frac{\mathrm{d}}{\mathrm{dt}}\left(x(t)-\sum_{i=1}^{m} c_{i}(t) x\left(t-r_{i}\right)\right)+\sum_{j=1}^{n} p_{j}(t) x\left(t-s_{j}\right)=0
$$

where the coefficients satisfy

(i) $0<r_{1} \leqslant r_{2} \leqslant \cdots \leqslant r_{m} ; 0<s_{1} \leqslant s_{2} \leqslant \cdots \leqslant s_{m}$;

(ii) $p_{j}(t)$ is continuous and $p_{j}(t) \geqslant q_{j} \geqslant 0, j=1,2, \cdots, n, t \in R, \sum_{j=1}^{n} q_{j}>0$.

(iii) $\quad c_{i}(t)$ is continuous and $0 \leqslant u_{i} \leqslant c_{i}(t), t \in R, \lim _{t \rightarrow \infty} c_{i}(t)=c_{i}, i=$ $1,2, \cdots, m$ and $0<\sum_{i=1}^{m} u_{i} \leqslant \sum_{i=1}^{m} c_{i}<1$

Then we have the following result:

LEMma 2.7. Assume that $y(t)$ is a nonoscillatory solution of (1.2). Then $\lim _{t \rightarrow \infty} y(t)=0$.

ProOF: The negative of a solution of (1.2) is again a solution of (1.2) and a nonoscillatory solution is an eventually positive or negative solution, so without loss of generality, we may assume that $y(t)$ is an eventually positive solution of (1.2). Then

$$
y(t)>0, y\left(t-r_{i}\right)>0, i=1,2, \cdots, m ; y\left(t-s_{j}\right)>0, j=1,2, \cdots, n
$$


for all $t \geqslant T_{1}$ for some $T_{1}>0$. Let

$$
z(t)=y(t)-\sum_{i=1}^{m} c_{i}(t) y\left(t-r_{i}\right)
$$

Then

$$
\frac{\mathrm{d}}{\mathrm{dt}} z(t)=-\sum_{j=1}^{n} p_{j}(t) y\left(t-s_{j}\right)<0, t \geqslant T_{1} .
$$

We see that $z(t)$ is a strictly monotone decreasing function for $t \geqslant T_{1}$, and so that $\lim _{t \rightarrow \infty} z(t)$ exists. If $\lim _{t \rightarrow \infty} z(t)=-\infty$, then $z(t)<0$ for all $t \geqslant T_{2}$ for some $T_{2} \geqslant T_{1}$. Hence

$$
y(t)<\sum_{i=1}^{m} c_{i}(t) y\left(t-r_{i}\right), t \geqslant T_{2} .
$$

Note that from conditions (iii) of (1.2),

$$
u_{i} \leqslant c_{i}(t)<c_{i}+\left(1-\sum_{i=1}^{m} c_{i}\right) /(2 m), i=1,2, \cdots, m
$$

for all $t \geqslant T_{3}$ for some $T_{3} \geqslant T_{2}$. It follows from (2.11) that

$$
y(t)<\sum_{i=1}^{m}\left(c_{i}+\left(1-\sum_{i=1}^{m} c_{i}\right) /(2 m)\right) y\left(t-r_{i}\right), t \geqslant T_{3}
$$

Let

$$
y\left(T_{3}-\tau\right)=\max _{1 \leqslant i \leqslant m}\left\{y\left(T_{3}-r_{i}\right)\right\}
$$

then

$y\left(T_{3}\right)<\sum_{i=1}^{m}\left(c_{i}+\left(1-\sum_{i=1}^{m} c_{i}\right) /(2 m)\right) y\left(T_{3}-\tau\right)=\left(1+\sum_{i=1}^{m} c_{i}\right) y\left(T_{3}-\tau\right) / 2, \tau \geqslant r_{1}>0$.

By iteration we have

$$
y\left(T_{3}+n \tau\right)<\left(\left(1+\sum_{i=1}^{m} c_{i}\right) / 2\right)^{n} y\left(T_{3}\right)
$$

which implies that $\lim _{n \rightarrow \infty} y\left(T_{3}+n \tau\right)=0$. Note that from $(2.9), \lim _{n \rightarrow \infty} z\left(T_{3}+n \tau\right)=0$. This contradicts the assumption that $\lim _{t \rightarrow \infty} z(t)=-\infty$. Hence

$$
\lim _{t \rightarrow \infty} z(t)=\alpha \in R \text {. }
$$


From (2.10), we have

$$
z\left(T_{3}\right)-\alpha=\int_{T_{3}}^{\infty} \sum_{j=1}^{n} p_{j}(s) y\left(s-s_{j}\right) d s \geqslant \sum_{j=1}^{n} q_{j} \int_{T_{3}}^{\infty} y\left(s-s_{j}\right) d s>0
$$

Thus $y \in L^{1}\left[T_{3}+s_{n}, \infty\right)$, and so that $z \in L^{1}\left[T_{3}, \infty\right)$. Hence $\alpha$ must equal 0 . Note that

$$
\begin{aligned}
& 0=\lim _{t \rightarrow \infty} z(t)=\lim _{t \rightarrow \infty} \inf z(t)=\lim _{t \rightarrow \infty} \inf y(t)-\left(\sum_{i=1}^{m} c_{i}\right) \lim _{t \rightarrow \infty} \sup y(t), \\
& 0=\lim _{t \rightarrow \infty} z(t)=\lim _{t \rightarrow \infty} \sup z(t)=\lim _{t \rightarrow \infty} \sup y(t)-\left(\sum_{i=1}^{m} c_{i}\right) \lim _{t \rightarrow \infty} \inf y(t),
\end{aligned}
$$

and then we have

$$
\lim _{t \rightarrow \infty} \inf y(t)=\left(\sum_{i=1}^{m} c_{i}\right) \lim _{t \rightarrow \infty} \sup y(t)=\left(\sum_{i=1}^{m} c_{i}\right)^{2} \lim _{t \rightarrow \infty} \inf y(t)
$$

which implies that

$$
\lim _{t \rightarrow \infty} \inf y(t)=\lim _{t \rightarrow \infty} \sup y(t)=0 \quad \text { or } \quad \infty
$$

Hence we have

$$
\lim _{t \rightarrow \infty} y(t)=0 \quad \text { or } \quad \infty .
$$

Note that $y \in L^{1}\left[T_{3}+s_{n}, \infty\right)$, and hence

$$
\lim _{t \rightarrow \infty} y(t)=0
$$

The proof is complete.

\section{Main Results}

In this section, we use the results in Section 2 to study sufficient conditions for oscillation of first order NDDEs (1.1) and (1.2). We obtain the following main results of this paper.

When $q_{k}=0$ where $1 \leqslant k \leqslant n$ or $u_{k}=0$ where $1 \leqslant k \leqslant m$ in the NDDEs (1.1) and (1.2), we list it (or them) in the following conditions and proofs of Theorem 3.1. and Theorem 3.2. for convenience. 
THEOREM 3.1. Assume that one of the following five conditions holds.
(a) $e \sum_{j=1}^{n} q_{j} s_{j} \geqslant 1-\sum_{i=1}^{m} c_{i} \exp \left(r_{i} \sum_{j=1}^{n} q_{j} /\left(1-\sum_{i=1}^{m} c_{i}\right)\right)$.
(b) $\sum_{j=1}^{n} q_{j} s_{j} \geqslant 1-\sum_{i=1}^{m} c_{i}$.
(c) $\sum_{j=1}^{n} q_{j} s_{j}<1-\sum_{i=1}^{m} c_{i}$, and
$e \sum_{j=1}^{n} q_{j} s_{j} \geqslant 1-\sum_{i=1}^{m} c_{i} \exp \left(r_{i} \sum_{j=1}^{n} q_{j} /\left(1-\sum_{i=1}^{m} c_{i}-\sum_{j=1}^{n} q_{j} s_{j}\right)\right)$.

(d) There exists a nonnegative integer $N$ satisfying

$$
e \sum_{j=1}^{n} q_{j} s_{j} \geqslant 1-\sum_{i=1}^{m} c_{i} \sum_{k=0}^{N}\left(\left(r_{i} \sum_{j=1}^{n} q_{j}\right) /\left(1-\sum_{i=1}^{m} c_{i}\right)\right)^{k} /(k !) .
$$

(e) $\quad \sum_{j=1}^{n} q_{j} s_{j}<1-\sum_{i=1}^{m} c_{i}$, and there exists a nonnegative integer $N$ satisfying

$$
e \sum_{j=1}^{n} q_{j} s_{j} \geqslant 1-\sum_{i=1}^{m} c_{i} \sum_{k=0}^{N}\left(\left(r_{i} \sum_{j=1}^{n} q_{j}\right) /\left(1-\sum_{i=1}^{m} c_{i}-\sum_{j=1}^{n} q_{j} s_{j}\right)\right)^{k} /(k !)
$$

Then all solutions of (1.1) are oscillatory.

PROOF: We shall show that the existence of a nonoscillatory solution of (1.1) leads to a contradiction. Suppose $y$ is a nonoscillatory solution of $(1.1)$; we suppose that $y(t)>0$ for all $t \geqslant T$ for some $T>0$ (If $y(t)<0$ eventually the procedure is similar.) It follows from lemma 2.6 that $\lim _{t \rightarrow \infty} y(t)=0$ and we have from (1.1) that

$$
\begin{aligned}
y(t) & =\sum_{i=1}^{m} c_{i} y\left(t-r_{i}\right)+\sum_{j=1}^{n} \int_{t}^{\infty} p_{j}(s) y\left(s-s_{j}\right) d s ; t \geqslant t_{0} \\
& \geqslant \sum_{i=1}^{m} c_{i} y\left(t-r_{i}\right)+\sum_{j=1}^{n} q_{j} \int_{t}^{\infty} y\left(s-s_{j}\right) d s ; t \geqslant t_{0}
\end{aligned}
$$

where $t_{0}=\max \left\{T+r_{m}, T+s_{n}\right\}$. It is not difficult to see from (3.1) that

$$
y(t) \geqslant c_{1} y\left(t-r_{1}\right), y(t)=y\left(t_{0}+n r_{1}+\tau\right) \geqslant \alpha \exp (-\mu t)
$$

for all $t \geqslant t_{0}$ where $n$ is a nonnegative integer and

$$
0 \leqslant \tau<r_{1} ; \mu=-\left(\ln \left(c_{1}\right)\right) / r_{1} ; \alpha=\exp \left(\mu t_{0}\right) \min _{t_{0} \leqslant t \leqslant t_{0}+r_{1}}\{y(t)\}
$$


Define the sequence $\left\{y_{k}(t)\right\}$ by

$$
\begin{aligned}
y_{0}(t) & \equiv y(t) \\
y_{k+1}(t) & \equiv \sum_{i=1}^{m} c_{i} y_{k}\left(t-r_{i}\right)+\sum_{j=1}^{n} q_{j} \int_{t}^{\infty} y_{k}\left(s-s_{j}\right) d s ; t \geqslant t_{0} .
\end{aligned}
$$

It follows from (3.1) and (3.4) that

$$
y_{1}(t)-y_{0}(t) \leqslant 0, y_{2}(t)-y_{1}(t) \leqslant 0, \cdots, y_{k+1}(t)-y_{k}(t) \leqslant 0 ; t \geqslant t_{0},
$$

which implies that

$$
y_{k+1}(t) \leqslant y_{k}(t) \leqslant \cdots \leqslant y_{1}(t) \leqslant y_{0}(t), t \geqslant t_{0} .
$$

Furthermore we have from (3.4) and (3.2) that

$$
y_{0}(t) \geqslant \alpha \exp (-\mu t), t \geqslant t_{0},
$$

and also one can derive using (3.3) that

$$
y_{1}(t) \geqslant \alpha \exp (-\mu t), y_{k+1}(t) \geqslant \alpha \exp (-\mu t), k=1,2, \cdots, t \geqslant t_{0} .
$$

Thus we have from (3.5) that

$$
\alpha \exp (-\mu t) \leqslant y_{k+1}(t) \leqslant y_{k}(t) \leqslant \cdots \leqslant y_{1}(t) \leqslant y_{0}, t \geqslant t_{0} .
$$

By Lebesgue's convergence theorem the pointwise limit of $\left\{y_{k}(t)\right\}$ exists and hence

$$
\alpha \exp (-\mu t) \leqslant y^{*}(t) \equiv \sum_{i=1}^{m} c_{i} y^{*}\left(t-r_{i}\right)+\sum_{j=1}^{n} q_{j} \int_{t}^{\infty} y^{*}\left(s-s_{j}\right) d s ; t \geqslant t_{0}
$$

where

$$
y^{*}(t)=\lim _{k \rightarrow \infty} y_{k}(t), t \geqslant t_{0}
$$

$y^{*}(t)$ is a nonoscillatory solution of the NDDE

$$
\frac{\mathrm{d}}{\mathrm{dt}}\left(x(t)-\sum_{i=1}^{i n} c_{i} x\left(t-r_{i}\right)\right)+\sum_{j=1}^{n} q_{j} x\left(t-s_{j}\right)=0, t \geqslant t_{0},
$$

where the coefficients satisfy

$$
\begin{array}{ll}
c_{i}>0, i=1,2, \cdots, m, \sum_{i=1}^{m} c_{i}<1 \quad \text { and } \quad 0<r_{1} \leqslant r_{2} \leqslant \cdots \leqslant r_{m} \\
q_{j} \geqslant 0, j=1,2, \cdots, n, \sum_{j=1}^{n} q_{j}>0 \quad \text { and } \quad 0<s_{1} \leqslant s_{2} \leqslant \cdots \leqslant s_{n} .
\end{array}
$$


(A) By Theorem 2.2, (3.6) cannot have a nonoscillatory solution when (a) holds. This contradiction proves all solutions of (1.1) are oscillatory.

(B) By Theorem 2.3 (i), (3.6) cannot have a nonoscillatory solution when (b) holds. This contradiction proves all solutions of (1.1) are oscillatory.

(C) By Theorem 2.3 (ii), (3.6) cannot have a nonoscillatory solution when (c) holds. This contradiction proves all solutions of (1.1) are oscillatory.

(D) By Theorem 2.4, (3.6) cannot have a nonoscillatory solution when (d) holds. This contradiction proves all solutions of (1.1) are oscillatory.

(E) By Theorem 2.5, (3.6) cannot have a nonoscillatory solution when (e) holds. This contradiction proves all solutions of (1.1) are oscillatory.

The proof is complete.

THEOREM 3.2. Assume that one of the following five conditions holds.

(a) $e \sum_{j=1}^{n} q_{j} s_{j} \geqslant 1-\sum_{i=1}^{m} u_{i} \exp \left(r_{i} \sum_{j=1}^{n} q_{j} /\left(1-\sum_{i=1}^{m} u_{i}\right)\right)$.

(b) $\sum_{j=1}^{n} q_{j} s_{j} \geqslant 1-\sum_{i=1}^{m} u_{i}$.

(c) $\sum_{j=1}^{n} q_{j} s_{j}<1-\sum_{i=1}^{m} u_{i}$, and

$$
e \sum_{j=1}^{n} q_{j} s_{j} \geqslant 1-\sum_{i=1}^{m} u_{i} \exp \left(r_{i} \sum_{j=1}^{n} q_{j} /\left(1-\sum_{i=1}^{m} u_{i}-\sum_{j=1}^{n} q_{j} s_{j}\right)\right)
$$

(d) There exists a nonnegative integer $N$ satisfying

$$
e \sum_{j=1}^{n} q_{j} s_{j} \geqslant 1-\sum_{i=1}^{m} u_{i} \sum_{k=0}^{N}\left(\left(r_{i} \sum_{j=1}^{n} q_{j}\right) /\left(1-\sum_{i=1}^{m} u_{i}\right)\right)^{k} /(k !) .
$$

(e) $\sum_{j=1}^{n} q_{j} s_{j}<1-\sum_{i=1}^{m} u_{i}$, and there exists a nonnegative integer $N$ satisfying

$$
e \sum_{j=1}^{n} q_{j} s_{j} \geqslant 1-\sum_{i=1}^{m} u_{i} \sum_{k=0}^{N}\left(\left(r_{i} \sum_{j=1}^{n} q_{j}\right) /\left(1-\sum_{i=1}^{m} u_{i}-\sum_{j=1}^{n} q_{j} s_{j}\right)\right)^{k} /(k !)
$$

Then all solutions of (1.2) are oscillatory.

Proof: We shall show that the existence of a nonoscillatory solution of (1.2) - leads to a contradiction. Suppose $y$ is a nonoscillatory solution of (1.2). Without loss of generality, we may suppose that $y(t)>0$ for all $t \geqslant T$ for some $T>0$. (If $y(t)<0$ 
eventually the procedure is similar.) It follows from lemma 2.7 that $\lim _{t \rightarrow \infty} y(t)=0$ and we have from (1.2) that

$$
\begin{aligned}
y(t) & =\sum_{i=1}^{m} c_{i}(t) y\left(t-r_{i}\right)+\sum_{j=1}^{n} \int_{t}^{\infty} p_{j}(s) y\left(s-s_{j}\right) d s ; t \geqslant t_{0} \\
& \geqslant \sum_{i=1}^{m} u_{i} y\left(t-r_{i}\right)+\sum_{j=1}^{n} q_{j} \int_{t}^{\infty} y\left(s-s_{j}\right) d s ; t \geqslant t_{0}
\end{aligned}
$$

where $t_{0}=\max \left\{T+r_{m}, T+s_{n}\right\}$. Without loss of generality, we may assume that $u_{1}>0$. It is not difficult to see from (3.9) that

$$
y(t) \geqslant u_{1} y\left(t-r_{1}\right), y(t)=y\left(t_{0}+n r_{1}+\tau\right) \geqslant \alpha \exp (-\mu t)
$$

for all $t \geqslant t_{0}$ where $n$ is a nonnegative integer and

$$
0 \leqslant \tau<r_{1} ; \mu=-\left(\ln \left(u_{1}\right)\right) / r_{1} ; \alpha=\exp \left(\mu t_{0}\right) \min _{t_{0} \leqslant t \leqslant t_{0}+r_{1}}\{y(t)\} .
$$

Define the sequence $\left\{y_{k}(t)\right\}$ by

$$
\begin{aligned}
y_{0}(t) & \equiv y(t) \\
y_{k+1}(t) & \equiv \sum_{i=1}^{m} u_{i} y_{k}\left(t-r_{i}\right)+\sum_{j=1}^{n} q_{j} \int_{t}^{\infty} y_{k}\left(s-s_{j}\right) d s ; t \geqslant t_{0} .
\end{aligned}
$$

It follows from (3.9) and (3.12) that

$$
y_{1}(t)-y_{0}(t) \leqslant 0, y_{2}(t)-y_{1}(t) \leqslant 0, \cdots, y_{k+1}(t)-y_{k}(t) \leqslant 0 ; t \geqslant t_{0},
$$

which implies that

$$
y_{k+1}(t) \leqslant y_{k}(t) \leqslant \cdots \leqslant y_{1}(t) \leqslant y_{0}(t), t \geqslant t_{0} .
$$

Furthermore we have from (3.12) and (3.10) that

$$
y_{0}(t) \geqslant \alpha \exp (-\mu t), t \geqslant t_{0}
$$

and also one can derive using (3.11) that

$$
y_{1}(t) \geqslant \alpha \exp (-\mu t), y_{k+1}(t) \geqslant \alpha \exp (-\mu t), k=1,2, \cdots, t \geqslant t_{0} .
$$

Thus we have from (3.13) that

$$
\alpha \exp (-\mu t) \leqslant y_{k+1}(t) \leqslant y_{k}(t) \leqslant \cdots \leqslant y_{1}(t) \leqslant y_{0}, t \geqslant t_{0} .
$$


By Lebesgue's convergence theorem the pointwise limit of $\left\{y_{k}(t)\right\}$ exists and hence

$$
\alpha \exp (-\mu t) \leqslant y^{*}(t) \equiv \sum_{i=1}^{m} u_{i} y^{*}\left(t-r_{i}\right)+\sum_{j=1}^{n} q_{j} \int_{t}^{\infty} y^{*}\left(s-s_{j}\right) d s ; t \geqslant t_{0}
$$

where

$$
y^{*}(t)=\lim _{k \rightarrow \infty} y_{k}(t), t \geqslant t_{0}
$$

$y^{*}(t)$ is a nonoscillatory solution of the NDDE

$$
\frac{\mathrm{d}}{\mathrm{dt}}\left(x(t)-\sum_{i=1}^{m} u_{i} x\left(t-r_{i}\right)\right)+\sum_{j=1}^{n} q_{j} x\left(t-s_{j}\right)=0, t \geqslant t_{0}
$$

where the coefficients satisfy

$u_{i} \geqslant 0, i=1,2, \cdots, m, 0<\sum_{i=1}^{m} u_{i}<1 \quad$ and $\quad 0<r_{1} \leqslant r_{2} \leqslant \cdots \leqslant r_{m}$

$q_{j} \geqslant 0, j=1,2, \cdots, n, \sum_{j=1}^{n} q_{j}>0 \quad$ and $\quad 0<s_{1} \leqslant s_{2} \leqslant \cdots \leqslant s_{n}$.

(A) By Theorem 2.2, (3.14) cannot have a nonoscillatory solution when (a) holds. This contradiction proves all solutions of (1.2) are oscillatory.

(B) By Theorem 2.3 (i), (3.14) cannot have a nonoscillatory solution when (b) holds. This contradiction proves all solutions of (1.2) are oscillatory.

(C) By Theorem 2.3 (ii), (3.14) cannot have a nonoscillatory solution when (c) holds. This contradiction proves all solutions of (1.2) are oscillatory.

(D) By Theorem 2.4, (3.14) cannot have a nonoscillatory solution when (d) holds. This contradiction proves all solutions of (1.2) are oscillatory.

(E) By Theorem 2.5, (3.14) cannot have a nonoscillatory solution when (e) holds. This contradiction proves all solutions of (1.2) are oscillatory.

The proof is complete.

REMARK. The sufficient conditions for oscillation of first order NDDEs (1.1) and (1.2) in this section include and are weaker than those of $[5,6]$, so the results of this section develop the results of $[\mathbf{5 , 6 ]}$. See Example 3, Example 4 and Example 5 in the Section 4 of this paper. 


\section{Examples}

In this section, we shall apply the results of this paper to some examples; furthermore we shall show from these examples that the sufficient conditions for oscillation of first order NDDEs which we obtained in this paper include and are in many cases weaker than those known and these sufficient conditons can be verified when a NDDE is given.

EXAMPLE 1. We consider the following NDDE

$$
\frac{\mathrm{d}}{\mathrm{dt}}\left(x(t)-\frac{1}{4} x\left(t-\frac{3}{2}\right)\right)+\frac{1}{2} x\left(t-\frac{1}{e}\right)=0,
$$

where

$$
m=n=1, c_{1}=\frac{1}{4}, r_{1}=\frac{3}{2}, p_{1}=\frac{1}{2}, s_{1}=\frac{1}{e}
$$

Note that

$$
p_{1} s_{1}=\frac{1}{2 e}<\frac{3}{4}=1-c_{1}, e p_{1} s_{1}=\frac{1}{2}=1-c_{1}\left(1+\frac{r_{1} p_{1}}{1-c_{1}}\right) .
$$

Then the condition

$$
p_{1} s_{1} e>1-c_{1} \quad \text { or } \quad p_{1} s_{1} e>1-c_{1}\left(1+\frac{r_{1} p_{1}}{1-c_{1}}\right)
$$

of $[2,3,4,5]$ does not hold. Hence the results of $[2,3,4,5]$ can not be applied to (4.1). But condition (2.7) (or (2.5), or (2.6), or (2.8)) holds when $m=n=1$. It follows from Theorem 2.4 (or Theorem 2.2, or Theorem 2.3, or Theorem 2.5) that all solutions of (4.1) are oscillatory.

EXAMPLE 2. We consider the following NDDE

$$
\frac{\mathrm{d}}{\mathrm{d} t}\left(x(t)-\frac{1}{4} x\left(t-\frac{1}{2}\right)-\frac{1}{8} x(t-1)\right)+\frac{1}{2} x\left(t-\frac{1}{e}\right)+\frac{1}{8} x\left(t-\frac{2}{e}\right)=0
$$

where

$$
\begin{aligned}
& m=n=2, c_{1}=\frac{1}{4}, c_{2}=\frac{1}{8} ; r_{1}=\frac{1}{2} \\
& r_{2}=1 ; p_{1}=\frac{1}{2}, p_{2}=\frac{1}{8} ; s_{1}=\frac{1}{e}, s_{2}=\frac{2}{e} .
\end{aligned}
$$

Note that

$$
\begin{aligned}
p_{1} s_{1}+p_{2} s_{2} & =\frac{3}{4 e}<\frac{5}{8}=1-c_{1}-c_{2} \\
e\left(p_{1} s_{1}+p_{2} s_{2}\right) & =\frac{3}{4}>\frac{3}{8}=1-c_{1}\left(1+\frac{r_{1}\left(p_{1}+p_{2}\right)}{1-c_{1}-c_{2}}\right)-c_{2}\left(1+\frac{r_{2}\left(p_{1}+p_{2}\right)}{1-c_{1}-c_{2}}\right) .
\end{aligned}
$$

Then condition (2.5) (or (2.6), or (2.7), or (2.8)) holds when $m=n=2$. It follows from Theorem 2.2 (or Theorem 2.3, or Theorem 2.4, or Theorem 2.5) that all solutions of (4.2) are oscillatory. 
EXAMPLE 3. We consider the following NDDE

$$
\frac{\mathrm{d}}{\mathrm{dt}}\left(x(t)-\frac{1}{4} x\left(t-\frac{3}{2}\right)\right)+\left(\frac{1}{2}+t\right) x\left(t-\frac{1}{e}\right)=0, t \geqslant 0,
$$

where

Note that

$$
m=n=1, c_{1}=\frac{1}{4}, r_{1}=\frac{3}{2}, p_{1}(t)=\frac{1}{2}+t \geqslant q_{1}=\frac{1}{2}, s_{1}=\frac{1}{e} .
$$

$$
e q_{1} s_{1}=\frac{1}{2}=1-c_{1}\left(1+\frac{r_{1} p_{1}}{1-c_{1}}\right) \text {. }
$$

Then condition (2.29) of [5] and $p_{1}(t)<w$ where $w$ is a positive constant do not hold, and hence [5, Theorem 2.3] and [6, Theorem 2.7] can not be applied to (4.3). But condition (d) of Theorem 3.1 holds when $m=n=1$, so it follows from Theorem 3.1 that all solutions of (4.3) are oscillatory.

EXAMPLE 4. We consider the following NDDE

$$
\frac{\mathrm{d}}{\mathrm{dt}}\left(x(t)-\left(\frac{1}{4}+\frac{1}{t}\right) x\left(t-\frac{3}{2}\right)\right)+\left(\frac{1}{2}+\frac{1}{t}\right) x\left(t-\frac{1}{e}\right)=0, t \geqslant 1,
$$

where

$$
m=n=1, c_{1}(t)=\frac{1}{4}+\frac{1}{t} \geqslant u_{1}=\frac{1}{4}, r_{1}=\frac{3}{2}, p_{1}(t)=\frac{1}{2}+\frac{1}{t} \geqslant q_{1}=\frac{1}{2}, s_{1}=\frac{1}{e} .
$$

Note that

$$
e q_{1} s_{1}=\frac{1}{2}=1-u_{1}\left(1+\frac{r_{1} q_{1}}{1-u_{1}}\right) .
$$

Then condition (d) of Theorem 3.2 holds when $m=n=1$, so it follows from Theorem 3.2 that all solutions of (4.4) are oscillatory.

EXAMPLE 5. We consider the following NDDE

$$
\begin{aligned}
\frac{\mathrm{d}}{\mathrm{dt}}\left(x(t)-\left(\frac{1}{4}+\frac{2}{t}\right) x\left(t-\frac{1}{2}\right)\right. & \left.-\left(\frac{1}{8}+\frac{1}{t}\right) x(t-1)\right) \\
+ & \frac{1}{2} x\left(t-\frac{1}{e}\right)+\left(\frac{1}{8}+2 t\right) x\left(t-\frac{2}{e}\right)=0, t>0
\end{aligned}
$$

where

$$
\begin{aligned}
& m=n=2, c_{1}(t)=\frac{1}{4}+\frac{2}{t} \geqslant u_{1}=\frac{1}{4}, c_{2}(t)=\frac{1}{8}+\frac{1}{t} \geqslant u_{2}=\frac{1}{8} ; r_{1}=\frac{1}{2}, \\
& r_{2}=1 ; p_{1}(t)=q_{1}=\frac{1}{2}, p_{2}(t)=\frac{1}{8}+2 t \geqslant q_{2}=\frac{1}{8} ; s_{1}=\frac{1}{e}, s_{2}=\frac{2}{e} .
\end{aligned}
$$

Note that

$$
e\left(q_{1} s_{1}+q_{2} s_{2}\right)=\frac{3}{4}>\frac{3}{8}=1-u_{1}\left(1+\frac{r_{1}\left(q_{1}+q_{2}\right)}{1-u_{1}-u_{2}}\right)-u_{2}\left(1+\frac{r_{2}\left(q_{1}+q_{2}\right)}{1-u_{1}-u_{2}}\right) .
$$

Then condition (d) (or (a), or (c), or (e)) of Theorem 3.2 holds when $m=n=2$, so it follows from Theorem 3.2 that all solutions of (4.5) are oscillatory. 


\section{REFERENCES}

[1] M.K. Grammatikopoulos, Y.G. Sficas and I.P. Stavroulakis, 'Necessary and sufficient conditions for oscillations of neutral equations with several coefficients', J. Differential Equations 76 (1988), 294-311.

[2] M.K. Grammatikopoulos, E.A. Grove and G. Ladas, 'Oscillations of first order neutral delay differential equations', J. Math. Anal. Appl. 120 (1986), 510-520.

[3] M.K. Grammatikopoulos, E. A. Grove and G. Ladas, 'Oscillation and asymptotic behaviour of first order neutral differential equations with deviating arguments', Appl. Anal. 22 (1986), 1-19.

[4] G. Ladas and Y.G. Sficas, 'Oscillations of neutral delay differential equations', Canad. Math. Bull. 29 (1986), 438-445.

[5] K. Gopalsamy and B.G. Zhang, 'Oscillation and nonoscillation in first order neutral differential equations', J. Math. Anal. Appl. 151 (1990), $42-57$.

[6] Jiang Ziwen, 'Oscillation of first order neutral delay differential equations', J. Math. Anal. Appl. 196 (1995), 800-813.

Department of Mathematics

Shandong Normal University

Jinan

Shandong 250014

People's Republic of China 